\title{
Facetten urbaner Strukturen
}

\author{
Angelika Krehl ${ }^{1}$ Andreas Klee ${ }^{2}$
}

Online publiziert: 28. August 2017

(C) Springer-Verlag GmbH Deutschland 2017

Urbane Strukturen - meist assoziiert mit Dichte, Kreativität und Lebendigkeit, aber auch mit Überfüllung, Lärm und schlechter Luft - sind der Ausgangspunkt für viele raumwissenschaftliche Untersuchungen. Gerade das Spannungsfeld zwischen Push- und Pull-Faktoren städtischen Lebens, und damit auch urbaner Strukturen, fasziniert seit Langem und bietet vielfältige Diskussionsansätze für Wissenschaft, Politik und Zivilgesellschaft.

Natürlich kann ein einzelnes Varia-Heft unserer Zeitschrift „Raumforschung und Raumordnung I Spatial Research and Planning" nicht das gesamte Spektrum möglicher Forschungsdesiderata und nötiger Handlungsbedarfe in Städten und Stadtregionen abdecken. Vielmehr werden in den hier versammelten Beiträgen einzelne Facetten urbaner Strukturen herausgegriffen, analysiert und eingeordnet. Im Fokus stehen die Themen Mobilität, grüne Infrastruktur, urbane ökonomische Resilienz, aber auch Kreativität als Ressource der Stadtentwicklung sowie Zentrale Orte als raumordnerisches Konzept. In der Zusammenschau aller Beiträge umfassen die angesprochenen Facetten urbaner Strukturen nicht nur inhaltliche und konzeptionelle Aspekte, sondern werden auch auf verschiedenen räumlichen Ebenen und in unterschiedlichen Untersuchungsdesigns betrachtet.

Dr. Angelika Krehl

angelika.krehl@ils-forschung.de

Prof. Dr. Andreas Klee

klee@arl-net.de

1 ILS - Institut für Landes- und Stadtentwicklungsforschung, Brüderweg 22-24, 44135 Dortmund, Deutschland

2 Akademie für Raumforschung und Landesplanung, Leibniz-Forum für Raumwissenschaften, Hohenzollernstraße 11, 30161 Hannover, Deutschland
So analysieren Heiko Rüger und Harun Sulak in ihrem Beitrag „Wochenendpendeln von Erwerbstätigen in Deutschland: Analysen mit den Mikrozensen 1991 bis 2012“ den Einfluss soziodemographischer sowie berufsund raumbezogener Merkmale auf die Wahrscheinlichkeit des Wochenendpendelns. Basierend auf dem kompletten Mikrozensusdatensatz weisen die Autoren zunächst eine kontinuierliche Zunahme der Wochenendpendler in den letzten Jahrzehnten nach. Des Weiteren können sie mithilfe regressionsanalytischer Verfahren einen Geschlechterunterschied bei den Faktoren des Wochenendpendelns herausarbeiten, der sich insbesondere auf die individuellen Lebensformen und den Berufsstatus der pendelnden Erwerbstätigen zurückführen lässt. Die umfangreiche Datengrundlage und die ausführlichen Analysen bestätigen bestehende Befunde, zeichnen aber darüber hinaus ein sehr differenziertes Bild des Wochenendpendelns in Deutschland. Im Fazit des Beitrags wird abschließend der ,typische“ Wochenendpendler skizziert.

Aus den vielfältigen Facetten des Urbanen greifen Henry Wüstemann und Jens Kolbe unter dem Titel „Der Einfluss städtischer Grünflächen auf die Immobilienpreise: Eine hedonische Analyse für die Stadt Berlin“ das Thema der grünen Infrastruktur heraus. Es darf nicht erst seit der Veröffentlichung des Hauptgutachtens des Wissenschaftlichen Beirats der Bundesregierung Globale Umweltveränderungen von 2016 (WBGU 2016a) als unbestritten gelten, dass Grünflächen und -strukturen eine wertvolle Grundlage für die nachhaltige Entwicklung von Städten darstellen. Ebenfalls muss festgehalten werden, dass der Nutzen urbaner Grünstrukturen sich auch in höheren Immobilienund Grundstückspreisen widerspiegeln kann. Die Analysen der Verfasser zeigen am Beispiel der Stadt Berlin einen positiven Einfluss städtischer Parkanlagen auf die Immobilienpreise auf. Sie weisen darüber hinaus nach, dass dieser 
positive Einfluss auch im Vergleich mit anderen urbanen Grünstrukturen bestehen bleibt - parknahe Immobilien sind relativ teurer. Damit betonen ihre Erkenntnisse die Bedeutung öffentlicher Güter und liefern wichtige Informationen zum Umfang der Kapitalisierung urbaner Grünflächen in Immobilienpreisen.

Auch Karsten Zimmermann, Christian Hundt, Christian Jütte und Carolin Pofalla befassen sich in ihrem Beitrag „Dortmund, Duisburg und die Rezession von 2008/2009 - Warum zwei Städte derselben Region ökonomisch unterschiedlich resilient sind" mit regionalökonomischen Fragestellungen. Sie suchen Antworten auf die Frage, wie die Städte des Ruhrgebiets auf die jüngste wirtschaftliche Krise 2008 reagierten - wie ökonomisch resilient sie also sind. Dazu analysierten sie, wie die aus dem Strukturwandel hervorgegangenen heutigen Branchenstrukturen, Akteur- und Governance-Zusammenhänge die Krisenfestigkeit der Ruhrgebietsstädte beeinflussen. Nun kann bei ,der Resilienz' noch nicht von einem etablierten und konzeptionell sauber gerahmten Konzept gesprochen werden, eine Tatsache, die auch die Autoren betonen. Deswegen wenden sie einen qualitativ-quantitativen Methodenmix an, bei dem sie mittels theoriegeleiteter Literaturanalyse und der Auswertung quantitativer Strukturindikatoren den Versuch unternahmen, Technische und Adaptive Resilienz mit einer evolutionären Perspektive zu verbinden. Stellvertretend für alle Großstädte des Ruhrgebiets wenden sie diesen Methodenmix in vergleichender Manier auf Dortmund und Duisburg an. Im Ergebnis dieser Untersuchungen attestieren sie der Stadt Dortmund tendenziell bessere ökonomische Resilienzeigenschaften als der Stadt Duisburg.

„Städte sind zunehmend auf innovative Ideen angewiesen, um im globalen Standortwettbewerb bestehen zu können. Diese Ideen entspringen einem kreativen Potenzial, welches sich nur mit Hilfe der kreativen Bewohner einer Stadt erschließen lässt.“ Mit diesen einleitenden Worten greift der Beitrag „Netzwerkstrukturen kreativ-urbaner Milieus in mittleren Großstädten“ von Katharina Bingel, Grit Leßmann, Jens Nußbaum und Brigitte Wotha ein hochaktuelles Thema im Rahmen der Debatte um die Kultur- und Kreativwirtschaft sowie deren Rolle als Motor für die wirtschaftliche Revitalisierung von europäischen Innenstädten auf. Eingebettet in die Analyse egozentrierter Beziehungsnetzwerke mehrerer ausgewählter Akteure werden Merkmale und Strukturen des kreativ-urbanen Milieus am Beispiel der Stadt Braunschweig herausgearbeitet. Der Beitrag erweitert damit die bisherigen Forschungen in Stadtplanung und Stadtsoziologie im Bereich der Kreativen Stadt, indem er einen expliziten Fokus auf mittlere Städte legt.

Der WBGU formuliert in seinem Factsheet 6 den „Bedarf an wissenschaftlich fundierten Handlungsempfehlungen“ und schreibt weiterhin: „Die Dringlichkeit der Handlungsnotwendigkeit steht oft im Kontrast zur langfristig angelegten Forschung“ (WBGU 2016b: 3). Mit dem Beitrag „Empirische Fundierung von Zentrale-Orte-Konzepten - Vorschlag für ein idealtypisches Vorgehen“" adressieren Thomas Terfrüchte, Stefan Greiving und Florian Flex ein raumwissenschaftlich ebenso wie gesellschaftlich relevantes Thema, das sich zudem passgenau in die vom WBGU beschriebenen Forschungs- und Beratungsdesiderata einfügt. Nicht zuletzt aufgrund ihrer umfangreichen gutachterlichen Tätigkeit in einigen Bundesländern sehen die Autoren die Notwendigkeit einer empirischen Fundierung von Zentrale-Orte-Konzepten. Anknüpfend an eine ausgezeichnete Darstellung und Analyse des aktuellen Diskussionsstandes über theoretische und methodische Fragen bei der Fortschreibung der Zentrale-Orte-Konzepte in den Landesraumordnungsprogrammen entwickeln sie ein iteratives Vorgehen zu deren Fortschreibung oder Neuaufstellung. Dabei konstatieren die Autoren allerdings auch, dass es die eine richtige Methode nicht gibt, sondern dass lediglich eine Reihe unterschiedlich gut geeigneter Vorgehensweisen zur Auswahl stehen. Wichtig sei, so das Fazit, dass sich die Plangeber über die Implikationen der jeweils gewählten Methode bewusst seien und damit auch eine Begründung liefern könnten, warum genau diese und nicht eine andere Methode angewendet wurde.

Wir hoffen, dass Sie in diesem Heft von „Raumforschung und Raumordnung I Spatial Research and Planning “ eigene, weitere Anknüpfungspunkte an die Diskussion und Erforschung unterschiedlicher Facetten urbaner Strukturen finden. Abschließend wünschen wir Ihnen eine gewinnbringende Lektüre.

\section{Literatur}

WBGU - Wissenschaftlicher Beirat der Bundesregierung Globale Umweltveränderungen (2016a): Der Umzug der Menschheit. Die transformative Kraft der Städte. Hauptgutachten 2016. Berlin.

WBGU - Wissenschaftlicher Beirat der Bundesregierung Globale Umweltveränderungen (2016b): Forschung für die Transformation der Städte zur Nachhaltigkeit. Berlin. = Factsheet 6 . 